\title{
Biodegradation of a magnesium alloy implant in the intercondylar femoral notch showed an appropriate response to the synovial membrane in a rabbit model in vivo
}

\author{
Marco Ezechieli', Julia Diekmannn ${ }^{1,2}$, Andreas Weizbauer ${ }^{1,2}$, Christoph Becher', \\ Elmar Willbold', Patrick Helmecke ${ }^{3}$, Arne Lucas ${ }^{4}$, Robert Schavan ${ }^{4}$ and \\ Henning Windhagen'
}

\begin{abstract}
Degradable magnesium alloys are promising biomaterials for orthopedic applications. The aim of this study was to evaluate the potential effects on both the synovial membrane (synovialis) and the synovial fluid (synovia) of the degradation products of a MgYREZr-pin implanted in the intercondylar femoral notch in a rabbit model. Thirty-six animals were randomized into two groups (MgYREZr or Ti6Al4V alloy) of 18 animals each. Each group was then divided into three subgroups with implantation periods of I, 4, and 12 weeks, with six animals in each subgroup. The initial inflammatory reaction caused by the surgical trauma declined after 12 weeks of implantation, and elucidated a progressive recovery of the synovial membrane. Compared with control Ti6Al4V pins, there were no significant differences between the groups. However, after 12 weeks, recovery of the synovial membrane was more advanced in the titanium group, in which $92 \%$ showed no signs of synovitis, than in the magnesium group. A cytotoxicity test with L929 cells and human osteoblasts (HOB) was also conducted, according to EN ISO I0993-5/I2, and no toxic leachable products were observed after $24 \mathrm{~h}$ of incubation. In conclusion, the MgYREZr alloy seems to be a suitable material for intra-articular degradable implants.
\end{abstract}

\section{Keywords}

Magnesium alloy, synovitis, degradable, cytotoxicity test, biocompatibility

\section{Introduction}

Surgical reconstruction of the anterior cruciate ligament (ACL) is a common orthopedic procedure. ${ }^{1}$ Interference screws made of metal, such as stainless steel or titanium, are often used for the fixation of the ACL. However, biodegradable implants may be preferable in order to avoid follow-up surgery for implant removal. Degradable screws manufactured of polymers such as poly-L-lactide or poly-DL-lactide-co-glycolide have been used clinically, but have displayed insufficient mechanical stability and the degradation products can produce adverse effects. ${ }^{2}$ Intraoperative and postoperative implant breakages of a resorbable polylactid acid screw have been reported, ${ }^{3,4}$ as well as implant displacement into the joint, ${ }^{5,6}$ joint swelling, ${ }^{7}$ and tunnel widening around the implant. ${ }^{8}$
In recent years, magnesium alloys have been investigated intensively as an alternative biodegradable material for orthopedic implants. ${ }^{9}$ Magnesium is the fourth most common cation in the human body, ${ }^{10}$

\footnotetext{
'Department of Orthopedic Surgery, Hannover Medical School, Hannover, Germany

${ }^{2}$ CrossBIT, Center for Biocompatibility and Implant-Immunology, Department of Orthopedic Surgery, Hannover Medical School, Hannover, Germany

${ }^{3}$ Institute of Production Engineering and Machine Tools (IFW), Leibniz Universität Hannover, Garbsen, Germany

${ }^{4}$ Syntellix AG, Schiffgraben II, Hannover, Germany
}

Corresponding author:

Marco Ezechieli, Department of Orthopedic Surgery, Hannover Medical School, Anna-von-Borries-Straße I-7, 30625 Hannover, Germany. Email: marco.ezechieli@ddh-gruppe.de 
and it is essential for many aspects of human physiology. ${ }^{11,12}$ The normal adult human body contains approximately $21-28 \mathrm{~g}$ of magnesium. ${ }^{13}$ The majority is located in bones $(60-65 \%)$, and only a small amount $(1-2 \%)$ is present in the human blood plasma. $^{12}$ The magnesium serum concentration of $0.65-1.05 \mathrm{mmol} / 1$ is regulated by the kidneys. ${ }^{13}$ About $70-80 \%$ of the magnesium appears in its cationic form $\left(\mathrm{Mg}^{2+}\right), 20-30 \%$ is associated with proteins, and the remainder is complexed with anions. ${ }^{13}$

From a technical point of view, magnesium possesses favorable mechanical properties (elastic modulus 41$45 \mathrm{GPa}$ and compressive yield strength $65-100 \mathrm{MPa}$ ) that are close to those of natural cortical bone and thus reduce the risk of undesirable stress-shielding phenomena. ${ }^{14,15}$ The most commonly used alloy elements are calcium, lithium, zinc, zirconium, aluminum, and rare earth elements (REE). ${ }^{9}$ According to DIN EN 1753, the magnesium alloy used in this study consists of MgYREZr, and is based on the alloy WE43. MgYREZr has showed good biological responses in both animal ${ }^{16}$ and clinical $^{17}$ studies. With respect to WE43, several studies have reported osteoconductive bioactivity. ${ }^{18,19}$ REE improve corrosion resistance and strength by solid solution strengthening and precipitation strengthening. ${ }^{20}$ Zirconium is typically used as a grain refinement agent in magnesium alloys, and contributes to strengthening. ${ }^{20}$

Magnesium and its alloys degrade continuously in body fluids, releasing $\mathrm{Mg}^{2+}$ ions and its alloying elements into the blood and the surrounding tissues. Chelated REE are excreted via the kidney. ${ }^{21}$ Little is known about the effects of the corrosion products of a degradable magnesium interference screw on the synovial membrane (synovialis) and the synovial fluid (synovia). In the present study, the effects of released ions and degradation products during the corrosion process were evaluated (i) in vitro with L929 and HOB cells, in accordance with EN ISO 10993-5/12; and (ii) in vivo with regard to the synovial membrane and fluid, using a magnesium alloy implant in the intercondylar femoral notch of the stifle joint of rabbits.

\section{Materials and methods}

\section{Study design}

The animal experiments were conducted under a protocol approved by the local ethics committee, in accordance with German federal animal welfare legislation (Approval No. 33.9-42502-04-12/0976) and with the National Institutes of Health Guide for the Care and Use of Laboratory Animals. We used 36 female New Zealand White rabbits (Charles River, Bad Kissingen, Germany), aged 6 months old, with a mean $( \pm \mathrm{SD})$ body weight of $3.8 \pm 0.2 \mathrm{~kg}$, which were randomized into two implant groups of 18 animals each. We used MgYREZr pins in the first group, and identically constructed titanium (Ti6Al4V) pins in the second group. Each group was subdivided into three subgroups, with implantation periods of 1,4 , and 12 weeks, with six animals in each subgroup. Blood samples were taken before surgery and at the end of the implantation periods to monitor possible influences of the corrosion products on the blood composition. The evaluation focused on the blood values of the liver and kidneys, and the concentration of magnesium in the serum. Blood samples were analyzed by a certified veterinary diagnostic laboratory (Laboklin, Bad Kissingen, Germany).

\section{Implants}

The pins (MgYREZr and Ti6Al4V) were produced by the Institute of Production Engineering and Machine Tools (IFW) of the Leibniz Universität Hannover. The implants had a total length of $9 \mathrm{~mm}$ and an external diameter of $2 \mathrm{~mm}$ and were cannulated with a diameter of $1 \mathrm{~mm}$. The magnesium alloy implants were made of a metallurgically processed magnesium alloy powder. This aluminum-free material (MgYREZr according to DIN EN 1753) is similar to WE43, and contains more than $90 \mathrm{wt} \%$ magnesium by weight. With an average grain size of less than $5 \mu \mathrm{m}$, this high-performance alloy exhibits an offset elastic limit of Rp $0.2>250 \mathrm{MPa}$, a tensile strength of $>275 \mathrm{MPa}$, and elongation at break of more than $10 \%$. The samples were sterilized prior to testing by gamma irradiation, using $25 \mathrm{kGy}$ of cobalt$60-\gamma$ radiation (BBF, Sterilization Services, Kernen, Germany).

\section{Surgical method}

Surgery was performed under general anesthesia, induced by intramuscular injection with ketamine (25 mg/kg; Ketanest; Albrecht, Aulendorf, Germany) and midazolam (5 mg/animal; Dormicum; CuraMED Pharma, Karlsruhe, Germany) and a subcutaneous dose of glycopyrroniumbromid $(0.1 \mathrm{mg} /$ animal; Robinul, Riemser Arzneimittel, Greifswald - Insel Riems, Germany). For analgesia, the rabbits were given meloxicam orally $(0.15 \mathrm{mg} / \mathrm{kg}$; Metacam; Boehringer Ingelheim, Germany) one day preoperatively, immediately preoperatively, and for the following two days. Additionally, they received preoperative buprenorphine subcutaneously $(0.15 \mathrm{mg} /$ animal; Temgesic; Essex Pharma, München, Germany). To perform the endotracheal intubation, each animal received an intravenous injection with propofol (initial $1 \mathrm{mg} / \mathrm{kg}$; PropofolLipuro $1 \%$; B. Braun Melsungen, Germany). 


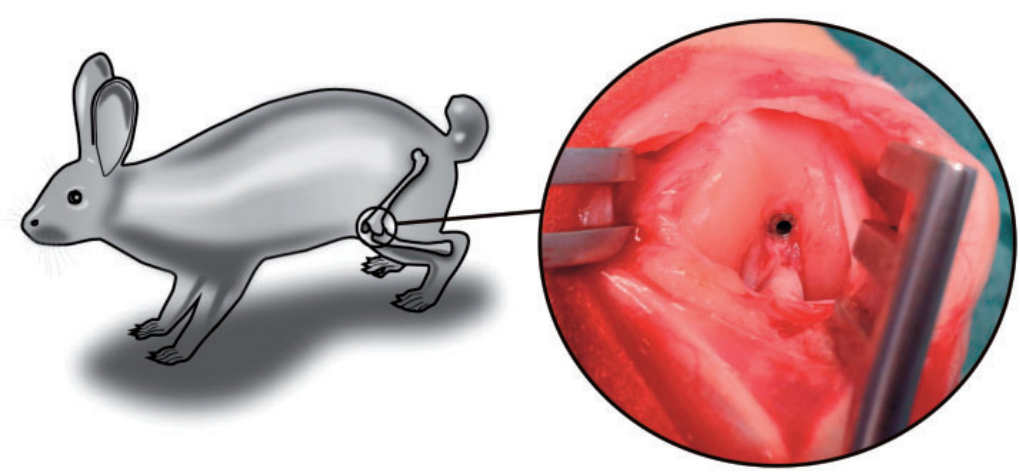

Figure I. Picture of implantation site. The MgYREZr and Ti6Al4V pins were implanted into the intercondylar femoral notch of rabbits.

Following the endotracheal intubation, anesthesia was maintained by isoflurane $(1.5-2.5 \%$ isoflurane by volume; Isofluran CP; CP-Pharma, Burgdorf, Germany) delivered in oxygen with flow rate of $1.0 \mathrm{l} / \mathrm{min}$. Infusion of Ringers lactate solution $(10 \mathrm{ml} / \mathrm{kg} / \mathrm{h})$ during the entire surgery ensured cardiovascular function.

In the left stifle joint, a medial parapatellar arthrotomy was performed to gain access to the femoral notch. Avoiding the cruciate ligaments, a drill hole with a diameter of $2 \mathrm{~mm}$ and a length of $8.8 \mathrm{~mm}$ was created with a hand-operated drill. The pins (magnesium in 18 animals; titanium in 18 animals) were inserted into the drill hole (Figure 1). The joint capsule, muscles, subcutis, and cutis were closed separately using absorbable suture material (Vicryl5-0; Ethicon, Norderstedt, Germany).

At the end of the investigation period, all animals were anaesthetized with ketamine $(25 \mathrm{mg} / \mathrm{kg})$ and midazolam ( $5 \mathrm{mg}$ /animal), and euthanized by an intravenous overdose of pentobarbital (release ad us. vet.; WDT, Garbsen, Germany).

\section{Postoperative treatment and radiological evaluation}

Postoperatively, the rabbits were allowed to move unrestricted in their cages, and they were observed daily for general condition, possible gas cavities, inflammation, joint effusion, or lameness. Antibiotic medication was given before surgery, and continued for four more days (enrofloxacin $10 \mathrm{mg} / \mathrm{kg}$ orally once daily; Baytril 2.5\% Bayer Animal Health, Leverkusen, Germany). Radiographs (anterior-posterior and lateral view) were taken immediately after surgery and after euthanasia to verify implant position and to detect possible formation of gas cavities.

\section{Histology of the synovial membrane}

After the implantation periods of 1, 4, and 12 weeks, the rabbits were euthanized. The synovial membranes were harvested at the medial and lateral locations of the joint and fixed in 3.5\% commercial formalin. Using standard procedures, the samples were then dehydrated in an increasing series of alcohol and embedded in paraffin wax using an automated embedding system to assure optimal quality. Using an SM 2000 R microtome (Leica Biosystems, Nußloch, Germany), $1 \mu \mathrm{m}$ thick sections were cut and mounted onto glass slides (Engelbrecht Medizin und Labortechnik, Edermünde, Germany). The sections were stained with haematoxylin and eosin (H\&E) according to established protocols.

\section{Histology of the synovial fluid}

The synovial fluid was processed for sectioning using a special method established by the Institute of Pathology of the University of Veterinary Medicine Hannover Foundation. Briefly, samples of the synovial fluid from both stifle joints of each euthanized rabbit were collected by arthrocentesis. The samples were transferred into plastic tubes containing a fixation solution of $5 \%$ glutaraldedyde and $1 \%$ sodium cacodylate. The tubes were then centrifuged for $15 \mathrm{~min}$ at $15,000 \mathrm{rpm}$ using a Universal $2 \mathrm{~S}$ centrifuge (Hettich, Tuttlingen, Germany). Using a Pasteur pipette, the pellets were harvested, transferred into $0.5 \mathrm{ml}$ eppendorf cups, and washed three times for $5 \mathrm{~min}$ each in sodium cacodylate buffer. After each wash, the pellets were centrifuged for $5 \mathrm{~min}$ at $1500 \mathrm{rpm}$. The pellets were post-fixed in a solution of $1 \%$ osmium tetroxide (Science Services, München, Germany) in sodium cacodylate buffer, and then washed and centrifuged again three times in cacodylate buffer. Finally, the buffer was removed with a Pasteur pipette and the pellets were carefully overlain with liquid 3\% Bacto Agar (BD Becton Dickinson, Heidelberg, Germany) which was heated to $100^{\circ} \mathrm{C}$ prior to use. Using a fine needle, the pellets and the Bacto Agar were carefully stirred. The tubes were incubated for $15 \mathrm{~min}$ in a water bath at 
Table I. Histopathological score of synovitis according to Krenn et al. $^{22}$ for evaluation of synovial membrane (synovalis).

\begin{tabular}{|c|c|}
\hline Evaluated parameter & Score \\
\hline \multicolumn{2}{|l|}{ (A) Hyperplasia / enlargement of synovial lining cell layer } \\
\hline - Absent & 0 \\
\hline - Slight enlargement (two or three cell layers); giant cells are very rare & I \\
\hline - Moderate enlargement (four to five cell layers); some giant cells or lymphocytes & 2 \\
\hline - Strong enlargement (more than six cell layers); giant cells; lymphocyes are frequent & 3 \\
\hline \multicolumn{2}{|l|}{ (B) Inflammatory infiltration } \\
\hline - Absent & 0 \\
\hline $\begin{array}{l}\text { - Slight inflammatory infiltration (diffusely locate single cells and small perivascular aggregates of } \\
\text { lymphocytes and/or plasma cells) }\end{array}$ & I \\
\hline $\begin{array}{l}\text { - Moderate inflammatory infiltration (perivascular and/or superficial lymphatic aggregates; small } \\
\text { lymphatic follicles without germinal center may be observed) }\end{array}$ & 2 \\
\hline $\begin{array}{l}\text { - Strong inflammatory infiltration (lymphatic follicles with germinal center and/or confluent } \\
\text { subsynovial lymphatic infiltration) }\end{array}$ & 3 \\
\hline \multicolumn{2}{|l|}{ (C) Activation of synovial stroma/pannus formation } \\
\hline - Absent & 0 \\
\hline $\begin{array}{l}\text { - Slightly synovial stroma activation (low cellularity with slight edem; slight fibrosis with some } \\
\text { fibroblasts; no giant cells) }\end{array}$ & I \\
\hline $\begin{array}{l}\text { - Moderate synovial stroma activation (moderate cellularity with a moderate density of fibro- } \\
\text { blasts and endothelial cells; giant cells may be detected) }\end{array}$ & 2 \\
\hline $\begin{array}{l}\text { - Strong synovial stroma activation (high cellularity with dense distribution of fibroblasts and } \\
\text { endothelial cells; giant cells are abundant) }\end{array}$ & 3 \\
\hline Maximum possible score for grading & 9 \\
\hline
\end{tabular}

$80^{\circ} \mathrm{C}$, and then for $5 \mathrm{~min}$ in a freezer at $-20^{\circ} \mathrm{C}$. The pellets were then carefully cut out of the tubes and transferred into standard biopsy-processing embedding cassettes lined with a filter paper. The samples were then dehydrated and embedded in paraffin wax using standard procedures. Using a Leica SM 2000 R microtome, $3-5 \mu \mathrm{m}$ thick sections were cut and mounted onto glass. The sections were stained with H\&E using established protocols.

\section{Histological evaluation of the synovitis score}

The histological evaluation of the synovial fluid and synovial membrane was determined using a semiquantitative score described by Krenn. ${ }^{22}$ For each animal, two samples of the synovial membrane were evaluated. The following morphological parameters of synovitis were selected for evaluation of the synovial membrane (Table 1):

(1) Hyperplasia of synovial lining layer;

(2) Activation of synovial stroma and

(3) Inflammatory infiltration.

Based on the change in the manifestation of each parameter (absent, slight, moderate, or strong), each parameter was scored in each sample, and the score of all parameters was summed to give a final score (Figure 2; Table 1) indicating the grade of synovitis (Table 2).

The photomicrographs of the synovial membrane sections were taken with an Axioskop 40 microscope combined with an AxioCam Mrc digital camera and AxioVision software (all Zeiss, Oberkochen, Germany).

Evaluation of the synovial fluid samples was conducted by the Department of Pathology, University of Veterinary Medicine, Hannover. The samples were assessed using a semiquantitative score (Figure 3; Table 3) developed by the aforementioned institute. The cells were counted in each sample per visual field at $400 \times$ magnification at three different locations. The distribution of cells in the sample is inhomogeneous due to the specific method of sample preparation. The mean value of each sample was determined and classified according to the semiquantitative score (Table 3 ).

\section{Preparation of MgYREZr extracts for cytotoxicity assay}

MgYREZr extracts were prepared in accordance with EN ISO 10993-5/12. The magnesium alloy samples were immersed under cell culture conditions for $24 \mathrm{~h}$ 

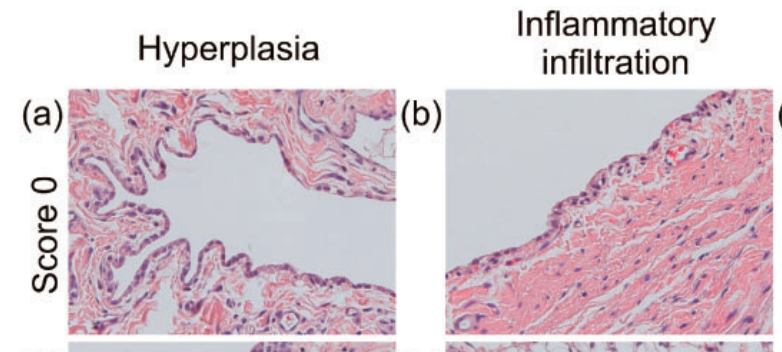

(e)
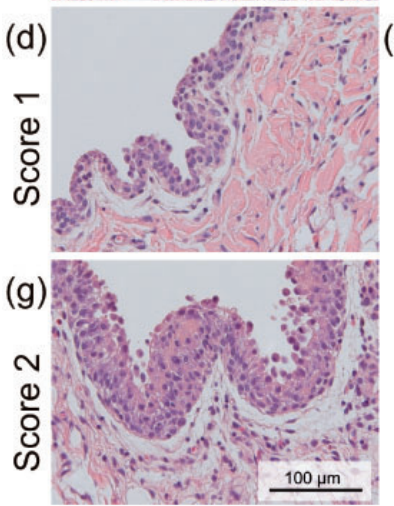



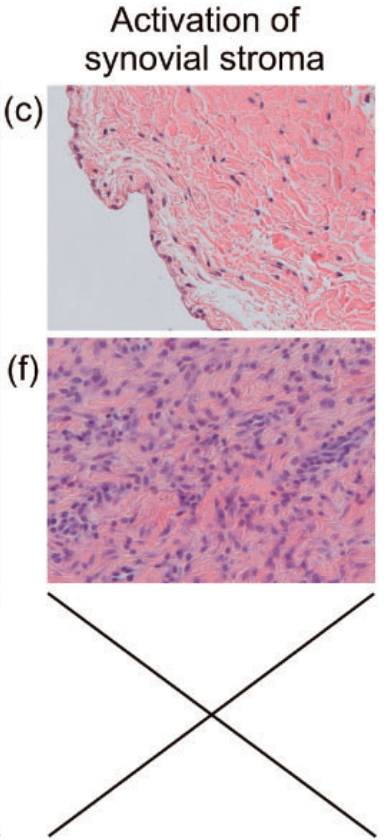

Figure 2. Hematoxylin and eosin staining of synovial membrane in its physiological conditions (a-c). The synovial lining cell layer consists physiologically of $\mathrm{I}-2$ cell layers (a), and these can increase up to $3-5$ layers in manifest synovitis (d,g). Slight inflammatory infiltration is classified as a score of I (e), and there is slight stroma activation with no giant cells (f).

Table 2. Classification of synovitis grade according to Krenn et al. $^{22}$ for evaluation of synovial membrane (synovalis).

\begin{tabular}{lll}
\hline Total score & Inflammatory grade & Evaluation \\
\hline $0-1$ & 0 & No synovitis \\
$2-3$ & 1 & Slight synovitis \\
$4-6$ & 2 & Moderate synovitis \\
$7-9$ & 3 & Strong synovitis \\
\hline
\end{tabular}

with a ratio of surface area to extraction medium volume of $1.25 \mathrm{~cm}^{2} / \mathrm{ml}$. High-density polyethylene film was used as a negative control $(\triangleq 100 \%$ viability), and a polyurethane film (zinc diethyldithiocarbamate) was used as a cytotoxic reference material (positive control) (both from Hatano Research Institute/Food and Drug Safety Center, Ochiai Hadano, Japan) with a ratio of surface area to extraction volume of $1.25 \mathrm{~cm}^{2} / \mathrm{ml}$. The extracts were filtered (pore size $0.22 \mu \mathrm{m}$ ) and the $\mathrm{pH}$ value and osmolality were then measured (SemiMicro Osmometer A0800, Knauer, Berlin, Germany).

\section{Evaluation of cell viability of MgYREZr extracts}

Mouse fibrosarcoma cells L929 were obtained from the Leibniz Institute DSMZ - German Collection of Microorganisms and Cell Cultures (Braunschweig, Germany). The cells were cultured in RPMI 1640 medium (Gibco/Life Technologies, Darmstadt,
Germany) plus $10 \%$ virus- and mycoplasma-free fetal bovine serum (PAN-Biotech, Aidenbach, Germany), and 1\% CellCultureGuard (AppliChem, Darmstadt, Germany) using standard cell culture conditions (90土2\% humidity, $37 \pm 1{ }^{\circ} \mathrm{C}, 5 \% \mathrm{CO}_{2}$, and $20 \% \mathrm{O}_{2}$ ).

Human primary osteoblasts (HOB; Promocell, Heidelberg, Germany) were cultured in HOB Growth Medium (Promocell, Heidelberg, Germany) supplemented with 1\% CellCultureGuard (AppliChem) under standard cell culture conditions and in accordance with the manufacturer's recommendations.

L929 cells were seeded into 96-well culture plates at a density of $1.5 \times 10^{4}$ cells $/ \mathrm{ml}$. HOB cells were seeded at a density of $3.9 \times 10^{4} \mathrm{cells} / \mathrm{ml}(0.2 \mathrm{ml} /$ well $)$. The cells were pre-cultivated for $24 \mathrm{~h}$, and then the complete medium was replaced by the sample extracts and the cells incubated for another $24 \mathrm{~h}$. The test was performed in eightfold parallel measurement. The viability of the cells was tested using an MTS assay (CellTiter 96 Aqueous One Solution Cell Proliferation Assay; Promega, Mannheim, Germany). Immediately before the test, the extracts were replaced with cell culture medium because tetrazolium salts interfere with magnesium. ${ }^{23}$ The extracts were incubated with the MTS salts for $2-3 \mathrm{~h}$. The soluble formazan product was quantified by measuring the absorbance using a $\mu$ Quant Microplate Spectrophotometer (BioTek Instruments, Bad Friedrichshall, Germany) at a wavelength of $490 \mathrm{~nm}$ (reference wavelength $650 \mathrm{~nm}$ ). In accordance with DIN EN ISO 10993-5, relative metabolic activity below $70 \%$ was regarded as cytotoxic. 


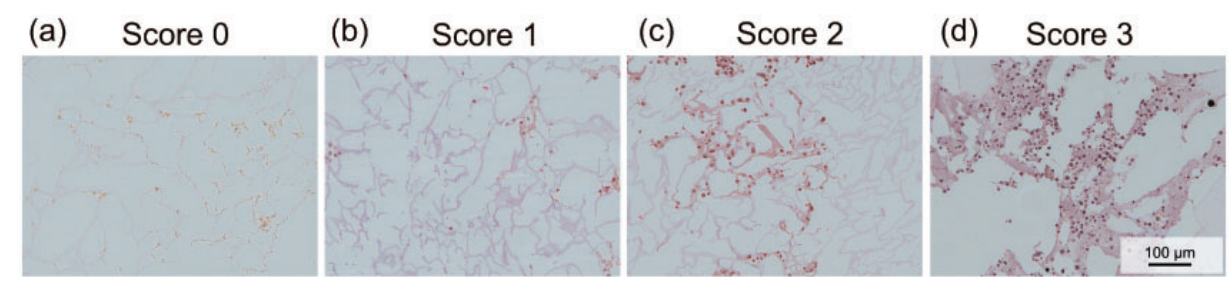

Figure 3. Hematoxylin and eosin staining of the synovial fluid. The synovitis of the synovial fluid was classified according to the number of cells (macrophage and cells type A) in each sample. The cells were counted in each sample per visual field at $400 \times$ magnification at three different locations. The distribution of cells in the sample is inhomogeneous due to the specific method of sample preparation.

Table 3. Semi-quantitative score for evaluation of histological synovial fluid (synovia).

\begin{tabular}{lll}
\hline $\begin{array}{l}\text { Number of cells } \\
\text { (macrophages or synovial } \\
\text { cells type A) }\end{array}$ & Grade of synovitis & Score \\
\hline$<2$ & No synovitis & 0 \\
$2-5$ & Slight synovitis & 1 \\
$6-10$ & Moderate synovitis & 2 \\
$>10$ & Strong synovitis & 3 \\
\hline
\end{tabular}

\section{Statistical analysis}

Statistical analysis was performed with the SPSS 20.0 software package (SPSS Inc., Chicago, IL, USA). Nonparametrical tests (Mann-Whitney $U$-test) were calculated to determine the differences between the implant groups (Mg and Ti). The Kruskal-Wallis test was calculated within the implant groups to determine significant differences between the implantation periods $(1,4$, and 12 weeks). If there were significant differences, a Mann-Whitney $U$-test was then performed. Statistical analysis of the blood values was performed using a $t$-test with a significance level of $p<0.05$.

\section{Results}

\section{Clinical observation of MgYREZr implants in vivo}

Clinically, all implants were well tolerated without any signs of inflammation, lameness, or evidence of subcutaneous gas cavities. The radiological outcome showed no excessive gas formation and no alterations of the bone structure (Figure 4). There were no significant differences in the blood values between the groups. The values of magnesium, creatinine, glutamic pyruvic transaminase (GPT), glutamic oxaloacetic transaminase (GOT), and white blood cell count (WBC) in the serum were not increased compared with normal levels. The serum levels of urea showed elevated concentrations before implantation and no further increases after implantation in both groups (Ti6Al4V and magnesium alloy).

\section{Moderate initial synovitis and progressive recovery of the synovial membrane after 12 weeks}

The synovial membrane showed mild inflammatory reactions after one and four weeks in both groups, without significant differences. Slight synovitis was seen one week after surgery in $75 \%$ of the rabbits in the magnesium group and $83 \%$ in the titanium group. After four weeks, about $8 \%$ of the samples showed evidence of moderate synovitis (score 2) in both the magnesium and titanium groups. At the end of the implantation period (12 weeks), there was no longer any evidence of moderate synovitis, and the main parts of the samples were assessed with score 0 (Figure 5). Differences between the magnesium and titanium groups after 12 weeks were not statistically significant. During the entire implantation period, there was no evidence of high-grade synovitis (score 3 ).

\section{No high-grade synovitis evaluated in the synovial fluid (synovia) after MgYREZr implantation}

One week after surgery, the magnesium and titanium groups both showed a synovitis score of 0-2 (Figure 6). Only one animal in the titanium group displayed highgrade synovitis (score 3). Four weeks after implantation, the titanium group showed either no synovitis or only slight synovitis, whereas in the magnesium group, a moderate synovitis was observed in 33\% of the animals. Twelve weeks after implantation, either slight synovitis or no synovitis was found in all animals of both groups. There were no significant differences between the groups.

\section{Cytotoxicity assay revealed no toxic leachable products of $\mathrm{MgYREZr}$}

The relative metabolic activity of HOB and L929 cells incubated with $100 \%$ extract were slightly decreased compared with the non-toxic negative control 

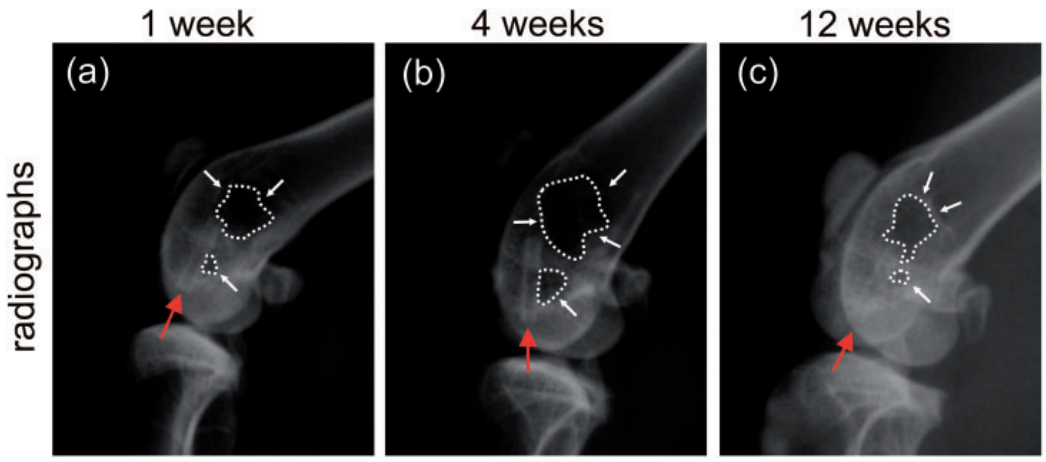

Figure 4. Radiographs of the implant in the femoral notch. The implant is marked by a red arrow, the evolved gas during degradation by white arrows, and the gas cavities by a dashed line. (a) Some gas was released at the upper part of the implant and accumulated in the medullary cavity. A small amount of gas was observed in direct contact with the implant. (b) The accumulation of gas was most pronounced after four weeks, and (c) was decreased after 12 weeks mainly due to diffusion processes and transported by the vascular system.

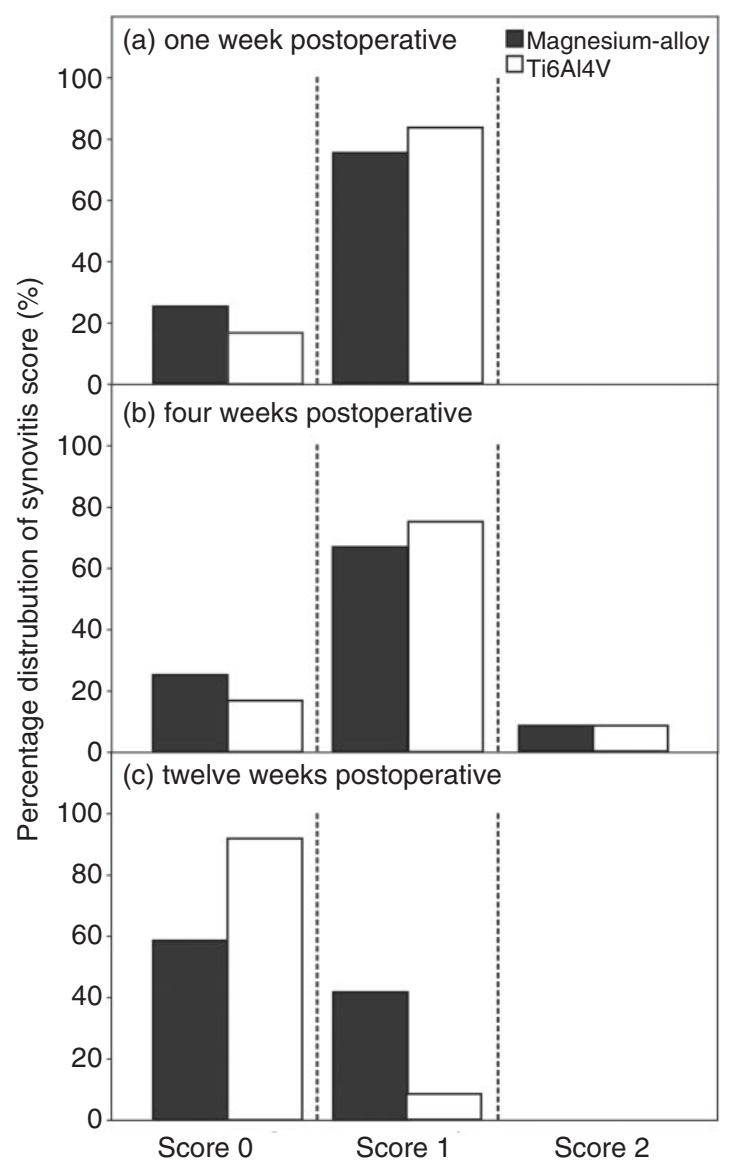

Figure 5. Histological evaluation of the synovial membrane (synovialis) according to Krenn's scoring system. After four weeks, there was some evidence of moderate synovitis (score 2) in both the magnesium alloy (black bars) and titanium (white bars) groups. The moderate initial synovitis regressed after 12 weeks in both groups.

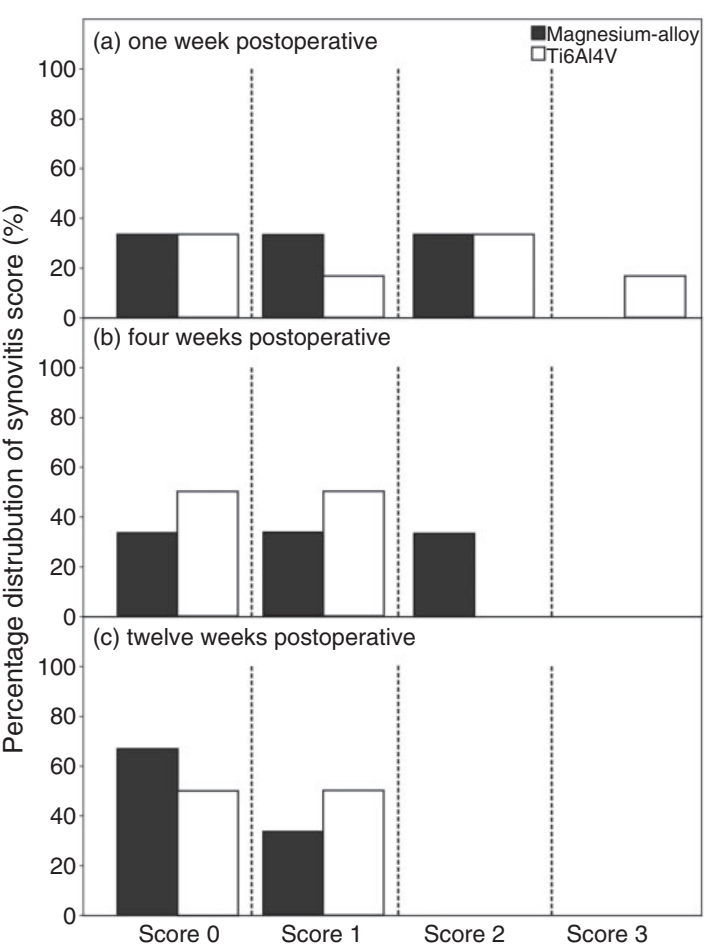

Figure 6. The synovial fluid (synovia) was evaluated by quantification of cells (macrophages or Type A synovial cells). The grade of synovitis decreases with implantation time in both the magnesium alloy (black bar) and titanium (white bar) groups. At 12 weeks after implantation, there was slight or no synovitis in both groups. 


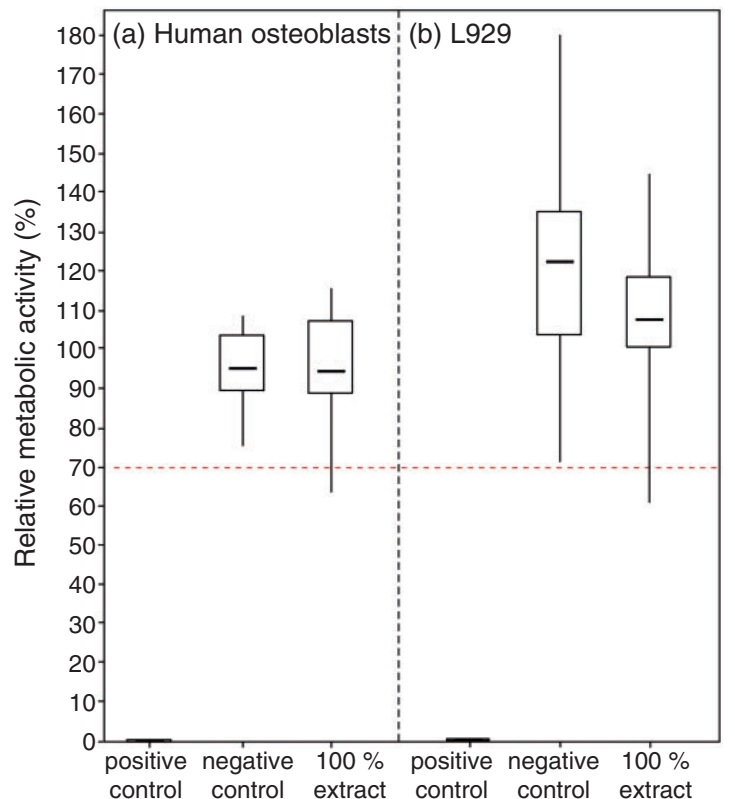

Figure 7. Relative metabolic activity of (a) human osteoblast (HOB) cells and (b) $\mathrm{L} 929$ cells after $24 \mathrm{~h}$ of incubation with extracts determined by MTS assay, shown as a box and whisker plot $(n=8)$. After exposure to $100 \%$ extract, both cell types displayed a relative metabolic activity above $70 \%$, which is considered non-cytotoxic. High-density polyethylene film was used as a negative control ( $\triangleq 100 \%$ viability) and a polyurethane film was used as a cytotoxic positive reference material.

(Figure 7). The viability rates of both cell types were above $70 \%$. The viability rate of the cytotoxic reference material was reduced to $0 \%$ in both setups.

The $\mathrm{pH}$ value of the $100 \%$ extract in the $\mathrm{HOB}$ medium was slightly increased compared with the aged growth medium. In RPMI $1640+10 \%$ FBS medium after $24 \mathrm{~h}$ of extraction, the $\mathrm{pH}$ value was nearly unchanged. In both media, the osmolality was decreased in the $100 \%$ extract compared with aged growth media (Figure 8).

\section{Discussion}

In this study, we investigated the effects of the degradation products of a specific magnesium alloy (MgYREZr) on the synovial membrane and the synovial fluid in a rabbit model in vivo, and on cell viability in an in vitro assay. As a control material, a commonly used titanium alloy (Ti6Al4V) was used, which is known for its good biocompatibility. ${ }^{24}$ The cannulated pin was implanted in the femoral "notch," protruding $1 \mathrm{~mm}$ so that the degradation ions had access to the joint without disturbing the joint function.

We hypothesized that the initial inflammatory reaction after drill surgery would decrease, followed by successive recovery of the synovial membrane without the appearance of severe inflammatory, foreign-body, or hypersensitivity reactions. The synovial membrane was histologically examined, and then rated using the validated, semiquantitative score of Krenn et al., ${ }^{22}$ which has shown a clear system of uniform diagnosis in other studies. ${ }^{25,26}$ One of the most common histological findings in inflammatory reactions of the synovial membrane is hyperplasia of the synovial lining cells. $^{27,28}$ The synovial lining cell layer consists physiologically of 1-2 cell layers, but in manifest synovitis, there can be $3-5$ cell layers. ${ }^{27,29}$ In our study, an enlarged lining cell layer in both groups was found. The synovitis was most pronounced after four weeks, and the grade of synovitis was decreased after 12 weeks. This recovery of the synovial membrane was clearer in the titanium group, with $92 \%$ showing no signs of synovitis after 12 weeks. The slower recovery of the synovial membranes in the magnesium group can be explained by the progressive degradation and release of corrosion products. The obvious hyperplasia of the lining cell layer ( $4-5$ cell layers) in the early postoperative period has been described by other authors. ${ }^{30}$ This hyperplasia decreased over the observation period, and a full recovery to physiological conditions was observed after around 30 days. ${ }^{27,30}$ In the present study, inflammatory reactions were still present after 30 days, which is consistent with the findings of Nio et al., ${ }^{29}$ who induced a post-traumatic synovitis by cutting the ACL. A study by Huebner et al. reported hyperplasia of the lining cell layer and defects in cartilage 9 and 52 weeks after drilling a hole in the femoral notch without using an implant. ${ }^{31}$ Those findings show that the operative trauma alone, without any implantation, leads to hyperplasia of the lining cell layer of the synovial membrane.

In addition to the histological evaluation of the synovial membrane, examination of the synovial fluid was performed, revealing signs of a chronic proliferative synovitis, and supporting the results of the synovial membrane examination, without significant differences between the groups. These findings show that the particles released during the degradation process of the magnesium alloy did not lead to any negative reactions in the synovial membrane, such as severe inflammation or foreign body reactions. No significant differences were found between the two groups, and regeneration of the synovial membrane was seen after 12 weeks.

Several studies have reported that non-degradable metallic implants, such as Ti6Al4V, lead to release of abrasive particles and ions from the base material. ${ }^{32,33}$ These may induce hypersensitivity reactions by activation of the immune system, ${ }^{32,34}$ and may potentially cause numerous local and systemic negative effects, such as inflammation, infection, pain, effusion, urticaria, or wound healing disorder. ${ }^{35,36}$ During the 


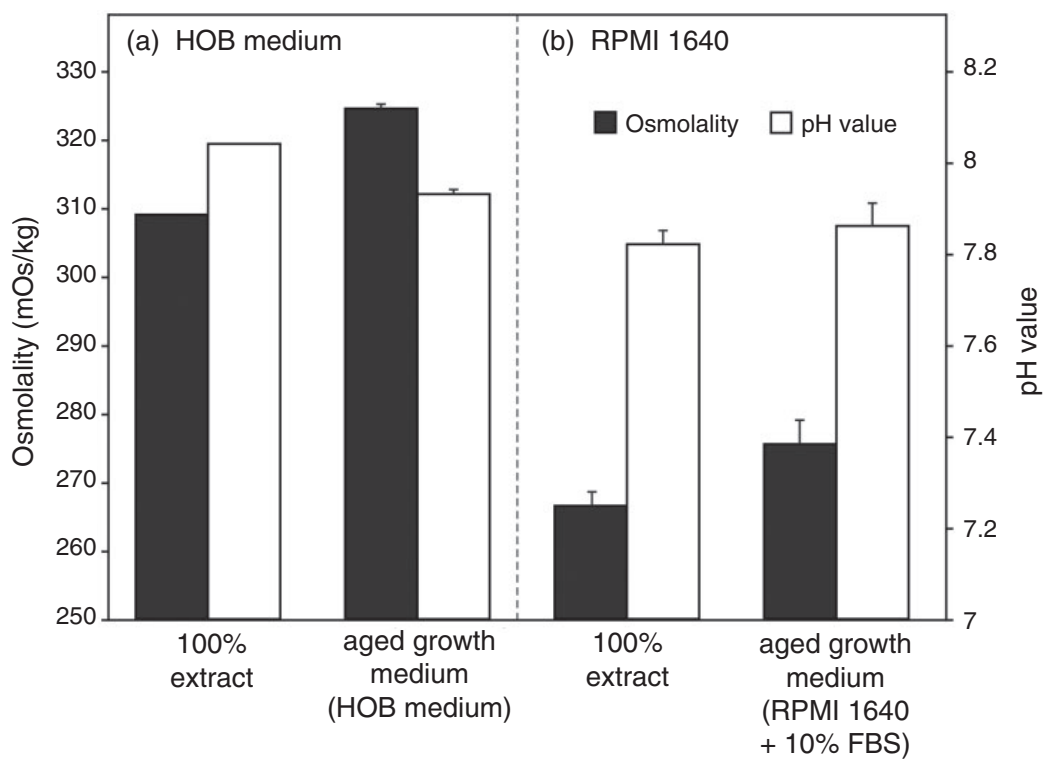

Figure 8. Osmolality and $\mathrm{pH}$ value of the MgYREZr extracts after $24 \mathrm{~h}$ of incubation in (a) Human Osteoblast Growth (HOB) Medium) and (b) RPMI 1640 plus 10\% fetal bovine serum (FBS). The osmolalitity was decreased in both media compared with aged growth medium. The $\mathrm{pH}$ value of the $100 \%$ extract of the $\mathrm{HOB}$ medium was increased.

degradation process of magnesium alloys, several degradation products are released, ${ }^{37-39}$ and may lead to the aforementioned inflammatory reactions. During the present study, the animals had no signs of insufficient wound healing or hypersensitivity reactions. These findings agree with those of Witte et al., who compared four different magnesium alloys (AZ31, AZ91, WE43, LAE442) with titanium (Ti6Al4V) and polymer (SRPLA96) ${ }^{40}$ No hypersensitivity reactions were described for any of the groups. ${ }^{40}$

Several studies have described foreign-body reactions and synovitis after implantation of degradable polymer implants in ACL reconstructive surgery. ${ }^{41-44}$ Barfod et al. and Fridén et al. described severe synovial reactions after intra-articular use of polyglycolide implants and of lactide-glycolide copolymer implants. ${ }^{41,42}$ A possible explanation for these reactions is that polymer particles attach to the synovial membrane and cause foreign-body reactions. ${ }^{45}$ However, these reactions were also observed in the implantation of non-degradable, synthetic implants in ACL reconstructive surgery. ${ }^{46-48}$ Several histological studies have detected phagocytes and multinucleated giant cells surrounding polymer particles. ${ }^{43,49}$ Of the 71 samples in the present study, seven (four titanium and three magnesium) had foreign body granulomas visible by the histological examination of the synovial membrane. We speculate that these were caused by the absorbable suture material and not by the degradation products of the magnesium alloy. Periarticular swelling in the lateral and dorsal parts of the knee were observed in four rabbits (two with titanium implants and two with magnesium implants), which decreased a few days after operation. No correlation between swelling and group (i.e. implant type) or time of implantation was found. This phenomenon has also been described by other authors. ${ }^{50}$

The indirect-cytotoxicity test is commonly applied to investigate the release of potential toxic particles of materials for medical applications in vitro. The released ions and degradation products from the magnesium alloy MgYREZr in the present study were non-toxic to L929 cells and HOB after $24 \mathrm{~h}$ of extract preparation. This test is valuable for initial screening of different materials prior to in vivo testing. Degradation rates in vitro differ from those found in in vivo studies. $^{39,51}$ Faster degradation rates of the material under in vitro conditions may lead to accumulation of various ions above toxic concentrations. In comparison with other tested magnesium alloys, MgY4 and RS66 reduced the cell viability in the $100 \%$ extract to under $70 \%{ }^{51,52}$ On the contrary, a previous study showed that the cell viability of osteoblasts was not reduced below $70 \%$ for a $\mathrm{Mg}$-10Dy alloy. ${ }^{53}$ The reduced relative metabolic activity of the $\mathrm{Mg}-10 \mathrm{Dy}$ alloy may be attributable to an increased $\mathrm{Mg}^{2+}$ content in the extract. ${ }^{53}$

In vitro experiments have shown increases in $\mathrm{pH}$ values during degradation of magnesium alloys because of the production of $\mathrm{OH}^{-} .9$ The optimal $\mathrm{pH}$ of $\mathrm{HOB}$ is within the range of 7.0-7.6. ${ }^{54}$ High $\mathrm{pH}$ values may have a negative effect on cell viability. In addition, solutions with high osmolality, due to high content of dissolved ions, may inhibit cell proliferation. ${ }^{55}$ In the current 
study, there was no marked change in either of these parameters in the in vitro extracts, and we therefore deduce that their influence on cell viability is minimal.

The magnesium alloy used in the present study contains an amount of REE besides zirconium. REE are often used in magnesium alloys to improve their mechanical (i.e. elasticity and ductility) and corrosive properties. $^{56}$ Numerous studies have shown good biocompatibility of alloys containing such REE. ${ }^{16,19,57}$ The magnesium alloy (MgYREZr) used in the present study has a similar composition to WE43, which has shown good biocompatibility, adequate corrosive characteristics, and slight effects on blood composition. ${ }^{16,18}$ Waizy et al. showed that the alloy used in this study had no negative effects on blood composition, and no pathological effects on the liver and the kidneys. ${ }^{16}$

Two recent in vivo studies reported the formation of gas cavities during the degradation of magnesium alloys. ${ }^{58,59}$ These accumulations of gas have been shown to disappear several weeks after implantation. ${ }^{37,60,61}$ Hydrogen gas $\left(\mathrm{H}_{2}\right)$ in particular may easily diffuse into capillaries and be transported by the vascular system. ${ }^{62}$ The low degradation rates of magnesium alloys result in small volumes of generated gas, but these gas formations are not clinically visible. $^{52,63,64}$

\section{Conclusions}

The present study investigated a MgYREZr alloy for use as potential intra-articular degradable implants. The results of the in vivo study with rabbits showed no significant differences between the titanium and the magnesium group. The synovitis was most pronounced after four weeks, and a recovery of the synovial membrane after 12 weeks in both groups was observed. It is supposed that the initial inflammatory reaction in the first weeks is mainly caused by the surgical trauma. The recovery of the synovial membrane was more advanced in the titanium group. To evaluate the cytotoxicity of this novel magnesium alloy, an in vitro test with L929 and HOB cells was conducted, in accordance with ISO 10993-5/12. No toxic leachable compounds were observed after $24 \mathrm{~h}$ of incubation. In conclusion, the MgYREZr alloy seems to be a suitable material for intra-articular use.

\section{Acknowledgements}

We thank Sylvie Bauer and Maren Hasper for excellent technical support. Furthermore, we thank Dr Tomas Correa for his valuable help with language accuracy and Dr Anneke Loos and Dr Christina Reufsteck for helpful discussions and excellent technical support. The authors acknowledge the financial support given by the German Research Foundation (DFG).

\section{Competing interests}

Mr Robert Schavan and Mr Arne Lucas are employed by the company Syntellix AG, Hannover, Germany. They have not influenced the collection of data or its interpretation. The other authors have no competing interests.

\section{References}

1. Pereira HMD, Correlo VM, Silva-Correia J, et al. Migration of 'bioabsorbable' screws in ACL repair. How much do we know? A systemic review. Knee Surg Sports Traumatol Arthrosc 2013; 21: 986-994.

2. Konan S and Haddad FS. A clinical review of bioabsorbable interference screw and their adverse effects in anterior cruciate ligament reconstruction surgers. Knee 2009; 16: 6-13.

3. Shafer BL and Simonian PT. Broken poly-L-lactic acid interference screw after ligament reconstruction. Arthroscopy 2002; 18: E35.

4. Werner A, Wild A, Ilg A, et al. Secondary intra-articular dislocation of a broken bioabsorbable interference screw after anterior cruciate ligament reconstruction. Knee Surg Sports Traumatol Arthrosc 2002; 10: 30-32.

5. Baums MH, Zelle BA, Schultz W, et al. Intraarticular migration of a broken biodegradable interference screw after anterior cruciate ligament reconstruction. Knee Surg Sports Traumatol Arthrosc 2006; 14: 865-868.

6. Krappel FA, Bauer E and Harland U. The migration of a BioScrew as a differential diagnosis of knee pain, locking after ACL reconstruction: a report of two cases. Arch Orthop Trauma Surg 2006; 126: 615-620.

7. Buelow JU, Siebold R and Ellermann A. A new bicortical tibial fixation technique in anterior cruciate ligament reconstruction with quadruple hamstring graft. Knee Surg Sports Traumatol Arthrosc 2000; 8: 218-225.

8. Emond CE, Woelber EB, Kurd SK, et al. A comparison of the results of anterior cruciate ligament reconstruction using bioabsorbable versus metal interference screws: a meta-analysis. J Bone Joint Surg Am 2011; 93: 572-580.

9. Waizy H, Seitz J-M, Reifenrath J, et al. Biodegradable magnesium implants for orthopedic applications. J Mater Sci 2013; 48: 39-50.

10. Fawcett WJ, Haxby EJ and Male DA. Magnesium: physiology and pharmacology. Br J Anaesth 1999; 83: 302-320.

11. Hartwig A. Role of magnesium in genomic stability. Mutat Res 2001; 475: 113-121.

12. Maguire ME and Cowan JA. Magnesium chemistry and biochemistry. BioMetals 2002; 15: 203-210.

13. Saris N-E, Mervaala E, Karppanen H, et al. Magnesium An update on physiological, clinical and analytical aspects. Clin Chim Acta 2000; 294: 1-26.

14. Nagels J, Stokdijk M and Rozing PM. Stress shielding and bone resorption in shoulder arthroplasty. $J$ Shoulder Elbow Surg 2003; 12: 35-39.

15. Staiger MP, Pietak AM, Huadmai J, et al. Magnesium and its alloys as orthopedic biomaterials: a review. Biomaterials 2006; 27: 1728-1734.

16. Waizy H, Diekmann J, Weizbauer A, et al. In vivo study of a biodegradable orthopedic screw (MgYREZr-alloy) 
in a rabbit model for up to 12 months. J Biomat Appl 2013; Epub ahead of print (10.1177/0885328212472215)).

17. Windhagen $H$, Radtke $K$, Weizbauer $A$, et al. Biodegradable magnesium-based screw clinically equivalent to titanium screw in hallux valgus surgery: short term results of the first prospective, randomized, controlled clinical pilot study. Biomed Eng Online 2013; 12: 62 .

18. Castellani C, Lindtner RA, Hausbrandt P, et al. Boneimplant interface strength and osseointegration: Biodegradable magnesium alloy versus standard titanium control. Acta Biomater 2011; 7: 432-440.

19. Witte F, Kaese V, Haferkamp H, et al. In vivo corrosion of four magnesium alloys and the associated bone response. Biomaterials 2005; 26: 3557-3563.

20. Witte F, Hort N, Vogt C, et al. Degradable biomaterials based on magnesium corrosion. Curr Opin Solid St Mater Sci 2008; 12: 63-72.

21. Hirano S and Suzuki KT. Exposure, metabolism, and toxicity of rare earths and related compounds. Environ Health Perspect 1996; 104: 85-95.

22. Krenn V, Morawietz L, Häupl T, et al. Grading of chronic synovitis - A histopathological grading system for molecular and diagnostic pathology. Pathol Res Pract 2002; 198: 317-325.

23. Fischer J, Prosenc MH, Wolff M, et al. Interference of magnesium corrosion with tetrazolium-based cytotoxicity assays. Acta Biomater 2010; 6: 1813-1823.

24. Pohler OE. Unalloyed titanium for implants in bone surgery. Injury 2000; 31: 7-13.

25. Krenn V, Morawietz L, Burmester GR, et al. Synovialitis score: histopathological grading system for chronic rheumatic and non-rheumatic synovialitis. $Z$ Rheumatol 2005; 64: 334-342.

26. Krenn V, Morawietz L, Burmester GR, et al. Synovitis score: discrimination between chronic low-grade and high-grade synovitis. Histopathology 2006; 49: 358-364.

27. Henderson B and Pettipher ER. The synovial lining cell: biology and pathobiology. Semin Arthritis Rheum 1985; 15: $1-32$.

28. Knöss P, Knöss M, Otto M, et al. Diagnostic spectrum of synovitis. Z Rheumatol 2008; 67: 8-16.

29. Nio J, Yokoyama A, Okumura M, et al. Three-dimensional ultrastructure of synoviocytes in the knee joint of rabbits and morphological changes in osteoarthritis model. Arch Histol Cytol 2002; 65: 189-200.

30. Kennedy A, Fearon U, Veale DJ, et al. Macrophages in synovial inflammation. Front Immunol 2011; 2: 52.

31. Huebner KD, Shrive NG and Frank CB. New surgical model of post-traumatic osteoarthritis: isolated intraarticular bone injury in the rabbit. J Orthop Res 2013; 31: 914-920.

32. Hallab NJ and Jacobs JJ. Biologic effects of implant debris. Bull NYU Hosp Jt Dis 2009; 67: 182-188.

33. Schmidt C, Ignatius AA and Claes LE. Proliferation and differentiation parameters of human osteoblasts on titanium and steel surfaces. J Biomed Mater Res 2001; 54: 209-215.

34. Cadosch D, Chan E, Gautschi OP, et al. Titanium IV ions induced human osteoclast differentiation and enhanced bone resorption in vitro. $J$ Biomed Mater Res A 2009; 91: 29-36.

35. Hallab N. Metal sensitivity in patients with orthopedic implants. J Clin Rheumatol 2001; 7: 215-218.

36. Thomas P, Schuh A, Ring J, et al. Orthopädisch-chirurgische Implantate und Allergien. Hautarzt 2008; 59: 220-229.

37. Hänzi AC, Gerber I, Schinhammer M, et al. On the in vitro and in vivo degradation performance and biological response of new biodegradable $\mathrm{Mg}-\mathrm{Y}-\mathrm{Zn}$ alloys. Acta Biomater 2010; 6: 1824-1833.

38. Persaud-Sharma D and McGoron A. Biodegradable Magnesium Alloys: A Review of Material Development and Applications. J Biomim Biomater Tissue Eng 2012; 12: 25-39.

39. Witte F, Fischer J, Nellesen J, et al. In vitro and in vivo corrosion measurements of magnesium alloys. Biomaterials 2006; 27: 1013-1018.

40. Witte F, Abeln I, Switzer E, et al. Evaluation of the skin sensitizing potential of biodegradable magnesium alloys. J Biomed Mater Res A 2008; 86: 1041-1047.

41. Barfod G and Svendsen RN. Synovitis of the knee after intraarticular fracture fixation with Biofix. Report of two cases. Acta Orthop Scand 1992; 63: 680-681.

42. Fridén T and Rydholm U. Severe aseptic synovitis of the knee after biodegradable internal fixation. A case report. Acta Orthop Scand 1992; 63: 94-97.

43. Hovis WD and Bucholz RW. Polyglycolide bioabsorbable screws in the treatment of ankle fractures. Foot Ankle Int 1997; 18: 128-131.

44. Lavery LA, Peterson JD, Pollack R, et al. Risk of complications of first metatarsal head osteotomies with biodegradable pin fixation: Biofix versus Orthosorb. J Foot Ankle Surg 1994; 33: 334-340.

45. Hoffmann R, Weiler A, Helling HJ, et al. Local foreignbody reactions to biodegradable implants A classification system. Unfallchirurg 1997; 100: 658-666.

46. Klein W and Jensen KU. Synovitis and artificial ligaments. Arthroscopy 8: 116-124. (21992).

47. Lukianov AV, Richmond JC, Barrett GR, et al. A multicenter study on the results of anterior cruciate ligament reconstruction using a Dacron ligament prosthesis in "salvage" cases. Am J Sports Med 1989; 17: 380-385.

48. Paulos LE, Rosenberg TD, Grewe SR, et al. The GORETEX anterior cruciate ligament prosthesis. A long-term followup. Am J Sports Med 1992; 20: 246-252.

49. Böstman OM and Pihlajamaki HK. Adverse tissue reactions to bioabsorbable fixation devices. Clin Orthop Relat Res 2000; 371: 216-227.

50. Konan S and Haddad FS. The unpredictable material properties of bioabsorbable PLC interference screws and their adverse effects in ACL reconstruction surgery. Knee Surg Sports Traumatol Arthrosc 2009; 17: 293-297.

51. Bobe K, Willbold E, Morgenthal I, et al. In vitro and in vivo evaluation of biodegradable, open-porous scaffolds made of sintered magnesium W4 short fibres. Acta Biomater 2013; Epub ahead of print (doi: 10.1016/ j.actbio.2013.03.035).

52. Willbold E, Kalla K, Bartsch I, et al. Biocompatibility of rapidly solidified magnesium alloy RS66 as a temporary 
biodegradable metal. Acta Biomater 2013; Epub ahead of print (doi: 10.1016/j.ctbio.2013.02.015).

53. Yang L, Hort N, Laipple D, et al. Element distribution in the corrosion layer and cytotoxicity of alloy $\mathrm{Mg}$ 10Dy during in vitro biodegradation. Acta Biomater 2013; Epub ahead of print (doi: 10.1016/ j.actbio.2012.10.001).

54. Kaysinger KK and Ramp WK. Extracellular pH modulates the activity of cultured human osteoblasts. $J$ Cell Biochem 1998; 68: 83-89.

55. Ha H, Yu MR, Choi HN, et al. Effects of conventional and new peritoneal dialysis solutions on human peritoneal metothelial cell viability and proliferation. Perit Dial Int 2000; 20: 10-18.

56. Hort N, Huang Y, Fechner D, et al. Magnesium alloys as implant materials-principles of property design for $\mathrm{Mg}$ RE alloys. Acta Biomater 2010; 6: 1714-1725.

57. Reifenrath J, Krause A, Bormann D, et al. Profound differences in the in-vivo-degradation and biocompatibility of two very similar rare-earth containing $\mathrm{Mg}$-alloys in a rabbit model. Mat -wiss u Werkstofftech 2010; 41: 1054-1061.

58. Fischerauer SF, Kraus T, Wu X, et al. In vivo degradation performance of micro-arc-oxidized magnesium implants: a micro-CT study in rats. Acta Biomater 2013; 9: 5411-5420.

59. Kraus T, Fischerauer SF, Hänzi AC, et al. Magnesium alloys for temporary implants in osteosynthesis: in vivo studies of their degradation and interaction with bone. Acta Biomater 2012; 8: 1230-1238.

60. Li Z, Gu X, Lou S, et al. The development of binary Mg$\mathrm{Ca}$ alloys for use as biodegradable materials within bone. Biomaterials 2008; 29: 1329-1344.

61. Zhang S, Zhang X, Zhao C, et al. Research on an Mg-Zn alloy as a degradable biomaterial. Acta Biomater 2010; 6: 626-640.

62. Kuhlmann J, Bartsch I, Willbold E, et al. Fast escape of hydrogen from gas cavities around corroding magnesium implants. Acta Biomater 2013; Epub ahead of print (doi: 10.1016/j.actbio.2012.10.008).

63. Huehnerschulte TA, Angrisani N, Rittershaus D, et al. In vivo corrosion of two novel magnesium alloys ZEK100 and AX30 and their mechanical suitability as biodegradable implants. Materials 2011; 4: 1144-1167.

64. Zhang E, Xu L, Yu G, et al. In vivo evaluation of biodegradable magnesium alloy bone implant in the first 6 months implantation. J Biomed Mater Res A 2009; 90: 882-893. 